\title{
Event-by-event Non-Rigid Data-driven PET Respiratory Motion Correction Methods: Comparison of Principal Component Analysis and Centroid of Distribution
}

Silin Ren ${ }^{1}$, Yihuan Lu$^{2}$, Ottavia Bertolli ${ }^{3}$, Kris Thielemans ${ }^{3}$, Richard E. Carson ${ }^{1,2}$

${ }^{1}$ Department of Biomedical Engineering, Yale University, New Haven, CT, USA

${ }^{2}$ Department of Radiology and Biomedical Imaging, Yale University, New Haven, CT, USA

${ }^{3}$ Institute of Nuclear Medicine, University College London, London, UK

For correspondence contact:

Silin Ren, Graduate student, Department of Biomedical Engineering, Yale University, PO Box 208048, New Haven, CT, USA, 06520-8048. E-mail: silin.ren@yale.edu

First author: Silin Ren, graduate student, Department of Biomedical Engineering, Yale University, PO Box 208048, New Haven, CT, USA, 06520-8048. E-mail: silin.ren@yale.edu 


\section{Abstract}

Respiratory motion is a major cause of degradation of PET image quality. Respiratory gating and motion correction can be performed to reduce the effects of respiratory motion; these methods require motion information, typically obtained from external tracking systems. Various groups have studied data-driven (DD) motion estimation methods. Recently, a data-driven respiratory motion estimation method was established by calculating the centroid of distribution (COD) of listmode events, which was then used with event-by-event respiratory motion correction (EBE$\mathrm{MC})$, and showed results comparable to those with an external motion tracking device. The EBEMC method only corrected for rigid motion, so that non-rigid components still contributed to motion-induced blurring. A nonrigid respiratory motion correction (NRMC) was later developed to overcome this problem, but was only evaluated using signal from an external monitor. Thus, it is desirable to further develop data-driven to achieve the best respiratory motion correction results.

We evaluated 2 data-driven respiratory motion detection methods, COD and Principal Component Analysis (PCA), by comparing the extracted motion trace to that acquired by the Anzai system in dynamic studies with two tracers. PCA was chosen as a preliminary study indicated that it produced stable results than other DD methods. We then developed and performed DD-EBE-NRMC using either COD- or PCA-derived respiratory motion information. Data-driven correction results were compared with Anzai-based results. For all tested studies, both COD and PCA showed good-to-excellent match with Anzai signals, with PCA showing a higher correlation with Anzai signals. The DD-EBE-NRMC results showed that both COD and PCA provide comparable image quality improvement as the Anzai-based correction. Although COD showed a lower correlation with Anzai than PCA, COD-based NRMC results are 
comparable to those of PCA, both of which showed great reduction in motion-induced blurring.

Key words: Respiratory Motion, Data-driven, Nonrigid Event-by-event Motion Correction 


\section{Introduction}

Respiratory motion produced substantial degradation in PET image quality. Respiratory gating and motion correction can be performed to reduce the effects of respiratory motion; these methods require motion information, typically acquired from external motion tracking systems. Because of the increased complexity and expense due to the use of external systems, these systems are rarely used in clinical practice and various groups have studied and proposed datadriven (DD) motion estimation methods; these can be organized into two main categories: imagebased, and raw data-based.

In Visvikis et al [1], respiratory motion was estimated from time activity curves obtained from regions-of-interest (ROIs) placed over the lung boundary on a series of reconstructed images (>150ms per frame) based on simulated data with the NCAT phantom. Another image-based method of Bundschuh et al [2] estimated respiratory motion by computing the image-based center-of-mass (COM) in Superior-Inferior (SI) direction of a volume of interest (VOI) over lesions in the lung or upper abdominal organs. The dynamic images were reconstructed with short time bins of 250, 500, and $750 \mathrm{~ms}$ per frame. Both methods require the reconstruction of a large number of dynamic images, which is computationally expensive and time-consuming.

Alternatively, multiple data-driven methods using PET raw data directly without image reconstruction have been proposed. In Schleyer et al [3-5], regions subject to respiratory motion identified by a spectral analysis method were first determined, then applied to rebinned sinogram data, each with a short time frame of $100 \mathrm{~ms}$. Respiratory motion signals were estimated by following the variations of total counts within each sinogram. In Kesner et al [6, 7], the sinogram region fluctuation method was proposed to generate $1 \mathrm{D}$ respiratory motion signals by combining 
information from the time activity curves from the entire sinogram. He et al [8] proposed the method of Geometric Sensitivity Gating, in which respiratory motion was estimated as variations in PET count rate over time utilizing the nonuniform sensitivity profile of PET scanner. A volume-of-interest (VOI) method was proposed by Buther et al [9] that applied a VOI over the motion-affected tumor in reconstructed image space. Then, the VOI was projected into sinogram space to determine the COM within this VOI based on raw data.

In an alternative to tracking total counts or determining COM, dimension reduction methods were also applied to raw data to extract respiratory motion. In Thielemans et al [10, 11], principal component analysis (PCA) was applied to a series of short time frame dynamic sinograms. The resulting 1D weight, with arbitrary scale factor, typically from the first component, was found to produce respiratory motion signals. Extending this approach further, Bertolli et al [12] proposed a method to automatically determine the sign of signals and applied the method to PET data with time-of-flight (TOF) information. With TOF information, better localization of individual events could be obtained. In a preliminary comparison study of different methods for data-driven respiratory gating, PCA was shown to have better and more stable results than other methods [11].

More recently, based on listmode data with TOF information, our group [13] proposed the datadriven respiratory motion estimation methods of COD (Centroid-Of-Distribution), in which for each event, the 3D coordinate is determined as the center of its TOF bin along its LOR. By averaging all event over each time frame (100ms), COD signals can be determined in three directions: superior-inferior (SI), anterior-posterior (AP), and left-right (LR), with SI and AP directions showing respiratory motions. Unlike previous data-driven methods which provide 1D motion signals based on total counts (COM in SI direction or PCA), COD also provides respiratory signals in the AP direction, because TOF information provides better localization of 
events in the transaxial plane (in AP and LR directions). In addition to data-driven respiratory gating, COD-based data-driven event-by-event respiratory motion correction (EBE-MC) was developed, and showed results comparable to those obtained with an external motion tracking device.

In [13], COD-based DD-EBE-MC only corrected for rigid motion, so that non-rigid components of respiratory motion contributed to motion-induced blurring. More recently, an EBE non-rigid respiratory motion correction (NRMC) method was established [14, 15], employing the respiratory motion trace acquired from an external device, and showed substantial improvement in image quality compared to the rigid correction. Thus, it is desirable to further establish DDEBE-NRMC to achieve the best respiratory motion correction results without using an external device.

In this work, we evaluated two data-driven respiratory motion detection methods, COD and PCA, by comparing the extracted respiratory motion signals to that acquired by the Anzai system (Anzai Medical, Tokyo, Japan) in dynamic studies with two tracers. Due to various tracer distribution and dynamics, DD methods do not always succeed in extracting good respiratory motion signals. Therefore, we first applied spectral analysis to the raw DD signals to identify studies with detectable DD signals. Then, we developed and performed DD-EBE-NRMC using either COD- or PCA-derived respiratory motion information on the selected studies. Data-driven correction results were compared with Anzai-based results; no-motion correction reconstructions were performed for comparison as well. 


\section{Methods}

\subsection{Centroid-Of-Distribution (COD)}

In the COD [13] method, for each listmode event $i$, given the detector pair information, the lineof-response (LOR) can be determined. Together with the TOF bin number, $\left(\mathrm{X}_{\mathrm{i}}, \mathrm{Y}_{\mathrm{i}}, \mathrm{Z}_{\mathrm{i}}\right)$ coordinates of each listmode event can be determined as the center of its TOF bin along the LOR, expressed in $\mathrm{mm}$ from the center of the FOV. Then, by averaging all events over each 100 -ms intervals COD signals are determined in three directions, with the SI direction $\left(\mathrm{C}_{\mathrm{z}}\right)$, showing the best respiratory motion compared with the gold standard of Anzai, and the AP direction $\left(\mathrm{C}_{\mathrm{y}}\right)$ showing weaker respiratory motion. In our previous COD study, we used both $\mathrm{C}_{\mathrm{z}}$ and $\mathrm{C}_{\mathrm{y}}$ for the EBE respiratory motion correction. However, both DD respiratory gating and DD-EBE-NRM, which will be introduced in this study, use $1 \mathrm{D}$ respiratory signals. Therefore, in this study, $\mathrm{C}_{z}$ will be used as the 1D respiratory input for DD-EBE-NRMC. Since the datasets were dynamic scans with changing tracer distributions, the slow kinetic drift of data-driven respiratory signals was removed by applying a sliding-average 120 s window, then, subtracting this moving average [16]. Then, to reduce noise, a low-pass filter with cutoff frequency of $0.5 \mathrm{~Hz}$ was applied.

\subsection{Principal Component Analysis (PCA)}

The PCA data-driven method [10] uses down-sampled sinograms rebinned from PET listmode data. Listmode data were binned into low-spatial-resolution dynamic sinograms (maintaining axial sampling) using STIR [17] without TOF information. The time duration for each sinogram was $100 \mathrm{~ms}$ (same as COD). After generating sinograms, principal component analysis was performed using MATLAB (MathWorks Inc., Natick, US). PCA generates several components, and the component corresponding to respiratory motion, which usually falls in the first two 
components, was selected by visual inspection. Due to the arbitrary scale factor of PCA-derived signals, the sign of the signal is sometimes reversed, so the sign was corrected through visual inspection. This process of component selection and sign determination can be fully automated as shown recently [12]. Elimination of slow kinetic drift and noise reduction were performed as described above for COD.

\subsection{Data Analysis}

Three dynamic datasets with two tracers were employed. All scans were acquired with the Siemens Biograph mCT PET/CT scanner (Siemens Medical Solutions, Knoxville, USA) with TOF information. Additionally, for all studies, the Anzai system (Anzai Medical, Tokyo, Japan) was used to acquire a respiratory trace at $50 \mathrm{~Hz}(20 \mathrm{~ms})$, which was rebinned to $100 \mathrm{~ms}$ per frame for comparison with COD and PCA signals.

\subsection{1. $\quad{ }^{18}$ F-FPDTBZ Pancreas Studies}

The first dataset contains studies with the tracer ${ }^{18}$ F-FPDTBZ [18], which is used for the imaging of pancreatic $\beta$-cell mass. In our previous work with COD and DD-rigid-EBE-MC [13], 10 pancreas studies were analyzed, showing good correlation between COD and Anzai, and good performance of data-driven respiratory motion correction. In this study, three subjects were randomly selected with COD and PCA signals determined over short periods: 10-20 min postinjection. The same 10min segment of data were employed for reconstructions with EBENRMC to compare Anzai signals to data-driven COD and PCA.

\subsection{2. $\quad{ }^{18}$ F-FDG Whole Body Oncology Studies}

The second dataset contains ${ }^{18} \mathrm{~F}-\mathrm{FDG}$ data from 9 whole-body oncology patients from University 
of Sydney. Listmode data were acquired over the chest or abdomen area for a 3-min period at 90 min postinjection. Note that the DD methods did not always succeed to extract a good respiratory signal. As discussed previously [13], studies with concentrated tracer uptake that moves with respiratory motion are more likely to provide robust data-driven signals. In this study, to first identify studies with data-driven signals, spectral analysis of the data-driven raw signals was performed.

First, a fast Fourier transform was applied to either COD or PCA signals (without low-pass filtering, but with removal of mean and standardization to unit variance) to determine the power spectrum. Respiratory cycles typically range from 2 to $10 \mathrm{~s}$, with an average cycle length of $6 \mathrm{~s}$. Therefore, if a DD method is successful, a significant fraction of the power should be found in the corresponding frequencies $(0.1$ to $0.5 \mathrm{~Hz})$. Therefore, to detect such a peak, Gaussian fitting with two components (gauss2 by MATLAB) was applied to the power spectrum:

$\mathrm{f}(\mathrm{x})=\mathrm{a} 1 \times \exp \left(-\left(\frac{x-b 1}{c 1}\right)^{2}\right)+\mathrm{a} 2 \times \exp \left(-\left(\frac{x-b 2}{c 2}\right)^{2}\right)$

If the peak location ( $b 1$ or $b 2$ ) fell in [0.1,0.5], and amplitude ( $a 1$ or $a 2$ ) was greater than an empirically determined cutoff, then it was determined that this trace contained respiratory motion signals. Three out of nine studies met the criteria set above based on both COD and PCA signals, providing detectable data-driven respiratory motion signals. Comparison between COD and PCA was performed with Anzai as gold standard. The same 3-min dataset was reconstructed with EBENRMC based on either COD, PCA, or Anzai signals, and compared with reconstructions without MC.

\subsection{3. $\quad{ }^{18}$ F-FDG Lung Oncology Studies}

The third dataset contains ${ }^{18} \mathrm{~F}-\mathrm{FDG}$ data from 10 lung cancer patients. Listmode data were 
acquired for $90 \mathrm{~min}$ and COD was determined from 60-70 min postinjection. To identify studies with data-driven respiratory motion signals, spectral analysis as described in 2.3 .2 was performed. Three out of ten studies were employed for the comparison between COD and PCA with Anzai signals, using EBE-NRMC reconstructions.

\subsection{Data-driven Non-Rigid Event-By-Event Respiratory Motion Correction}

In EBE-NRMC [14, 15], first, respiratory phase-gated reconstructions are performed, with one gate chosen to be the reference gate. Then, non-rigid registrations [19] are performed between reconstructions of the reference gate and the remaining gates. The resulting voxel-wise motion vectors are then used as the inter-gate motion estimates. A linear correlation is then built between each voxel's motion estimates and the mean displacement in each gate. The correlation can then be applied back using the continuous respiratory motion trace to generate continuous respiratory motion for each voxel at each time point. This information is subsequently incorporated into the EBE-NRMC by MOLAR. In this study, for each dataset, the entire analysis was performed with 3 respiratory tracers: Anzai, COD, and PCA.

When reconstructing respiratory gated images for nonrigid registrations in EBE-NRMC, for ${ }^{18} \mathrm{~F}$ FPDTBZ pancreas studies and ${ }^{18} \mathrm{~F}-\mathrm{FDG}$ whole body studies over the liver, no attenuation correction was performed to avoid artefacts near the diaphragm due to PET-CT mismatch. However, this approach was not successful for the ${ }^{18}$ F-FDG lung studies, where contrast between tissue, lesions, and air was lost or reduced without attenuation correction. For these studies, we employed our recently developed approach [15] for inter-gate registration using MLACF (maximum likelihood activity and attenuation correction factors estimation) [20]. 


\section{Results}

\subsection{COD, PCA, and Anzai Comparison}

\subsection{1. $\quad{ }^{18}$ F-FPDTBZ Pancreas Studies}

For the three subjects analyzed, COD, and PCA tracers are overlaid with Anzai in Figure 1, after removal of the mean, and standardization to unit variance. Visually, both COD and PCA showed very good correlation with Anzai traces in terms of both timing and motion amplitude, although the COD trace in subject 1 showed some differences. For quantitative comparison, correlation coefficients between pairs of these signals were calculated, as shown in Table 1. In two of three subjects, PCA showed higher correlation coefficients with Anzai than COD, with the lowest COD vs. Anzai correlation of 0.76, i.e., still reasonably good. All three subjects showed PCA vs. Anzai correlation $\geq 0.9$. Additionally, the correlation between COD and PCA also provided excellent match, with correlations $\geq 0.9$. 

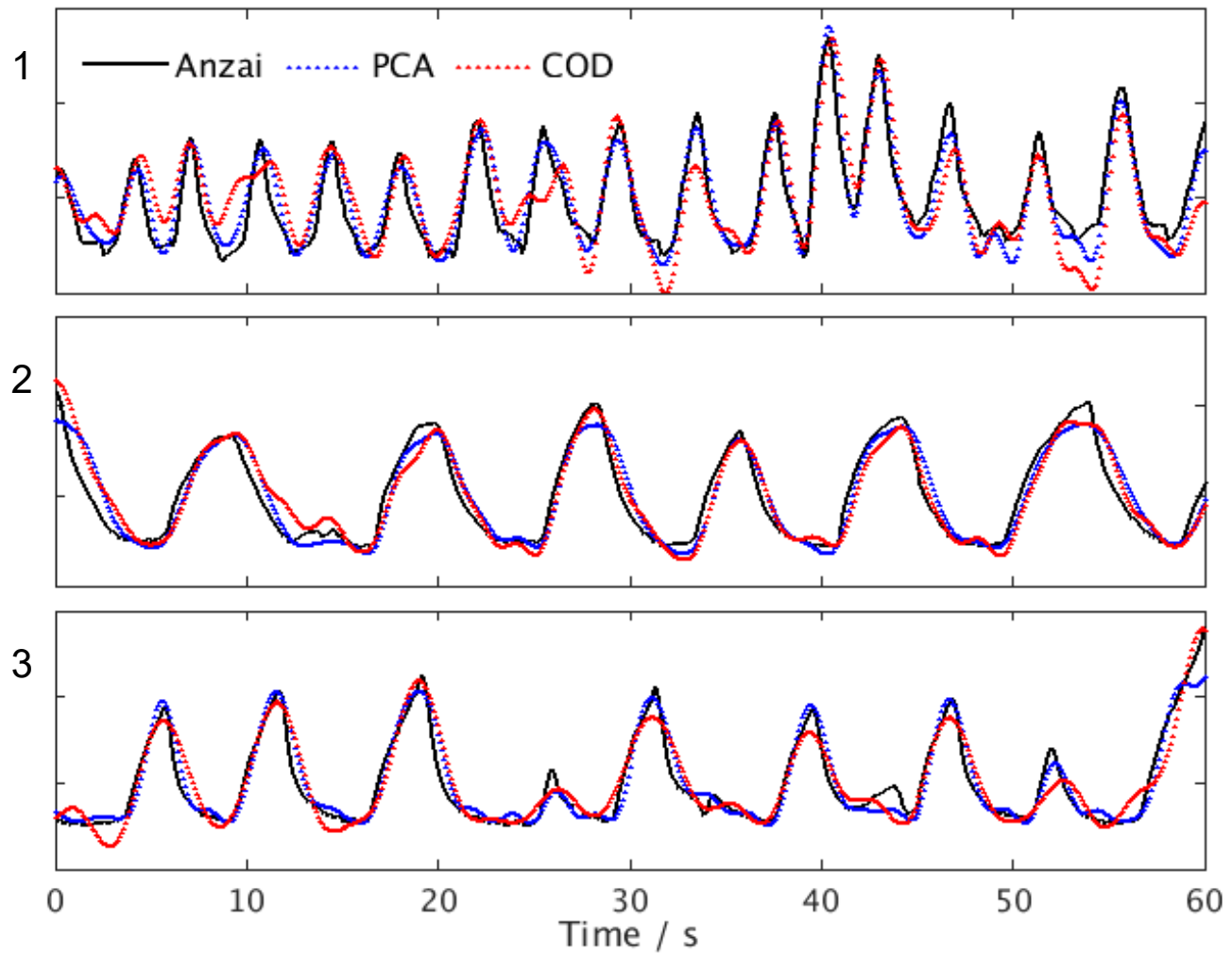

Figure 1 Overlaid Anzai (black), PCA (blue), and COD (red) traces for three ${ }^{18}$ F-FPDTBZ pancreas studies, displaying $1 \mathrm{~min}$ of data starting $12 \mathrm{~min}$ post-injection. Correlation coefficients of PCA vs. Anzai shown in this figure were: 0.92, 0.93, and 0.95; COD vs Anzai: 0.78, 0.92, and 0.93 ; PCA vs. COD: $0.88,0.96$, and 0.92 .

Table 1 Correlation coefficients between COD-Anzai, PCA-Anzai, and COD-PCA for 10-20 min post-injection of three ${ }^{18} \mathrm{~F}$-FPDTBZ studies, 0 -3min post-injection of three ${ }^{18} \mathrm{~F}$-FDG whole body (WB) studies, and 50-60min post-injection of ${ }^{18} \mathrm{~F}-\mathrm{FDG}$ lung cancer studies.

\begin{tabular}{|l|l|l|l|l|l|l|l|l|l|}
\hline & \multicolumn{4}{|l|}{${ }^{18}$ F-FPDTBZ } & \multicolumn{4}{l|}{${ }^{18}$ F-FDG WB } & \multicolumn{3}{l|}{${ }^{18}$ F-FDG Lung } \\
\cline { 2 - 11 } & 1 & 2 & 3 & 1 & 2 & 3 & 1 & 2 & 3 \\
\hline COD Vs Anzai & 0.76 & 0.95 & 0.85 & 0.83 & 0.63 & 0.92 & 0.72 & 0.84 & 0.19 \\
\hline PCA Vs Anzai & 0.90 & 0.93 & 0.91 & 0.94 & 0.71 & 0.90 & 0.87 & 0.86 & 0.16 \\
\hline COD Vs PCA & 0.91 & 0.98 & 0.95 & 0.85 & 0.91 & 0.93 & 0.83 & 0.78 & 0.76 \\
\hline
\end{tabular}




\subsection{2. $\quad{ }^{18}$ F-FDG Whole Body Oncology Studies}

Spectral analysis was applied to these nine studies. Three studies were identified to contain respiratory signals in data-driven traces, both from COD and PCA signals. First, for COD, Figure 2 shows one example subject, which was found to contain respiratory motion in its COD trace. The raw COD trace is shown in a), with the power spectrum in b), and the fitted Gaussian model in c). Visually from a), before low-pass filtering, respiratory motion can be observed. In its corresponding power spectrum, a large peak can be observed near $0.2 \mathrm{~Hz}$. After applying Gaussian fitting, the power spectrum was fitted and met the pre-defined thresholds for presence of respiratory signals.

a)

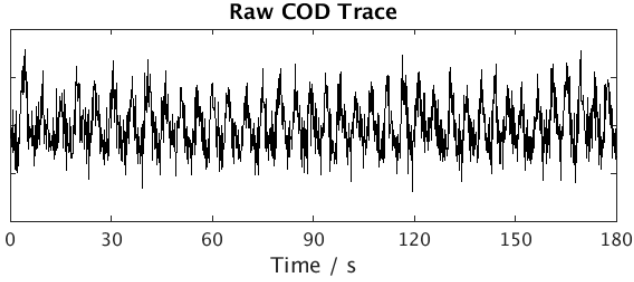

b)

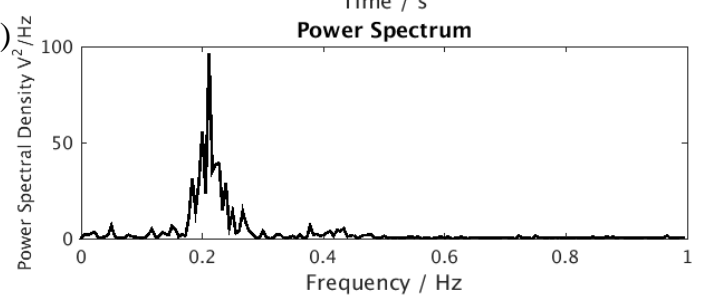

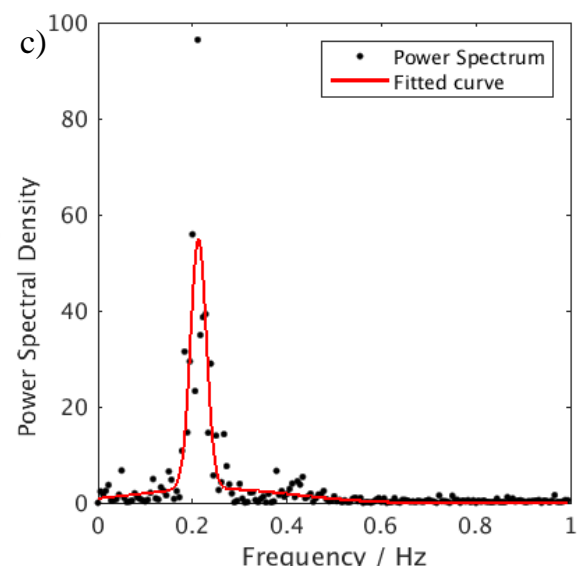

Figure 2 One example FDG subject which was identified to contain respiratory signals in COD: a) raw COD 3-min tracer, b) Power spectrum of COD, and c) Power spectrum with fitted Gaussian model.

Correspondingly, for subjects without respiratory signals in data-driven traces, a significant fraction peak could not be detected within $0.1-0.5 \mathrm{~Hz}$. One example subject without respiratory motion in COD is shown in Figure 3. Note the different scale in plots b) and c) than in Figure 2. 
a)

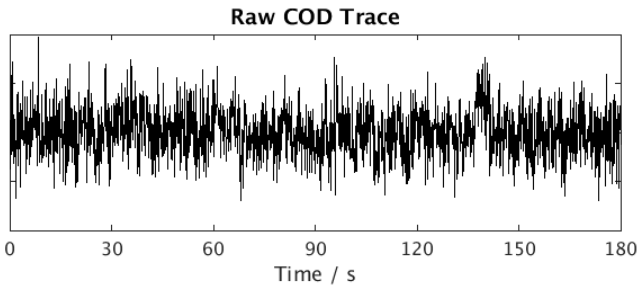

b)

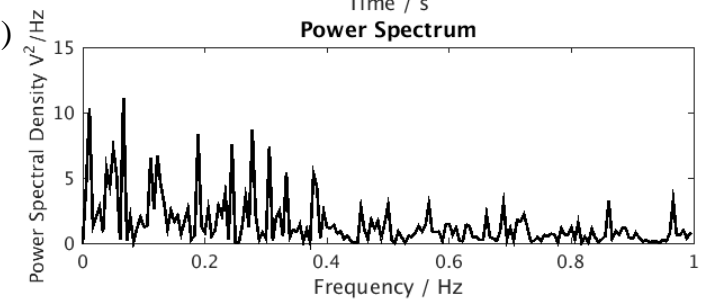

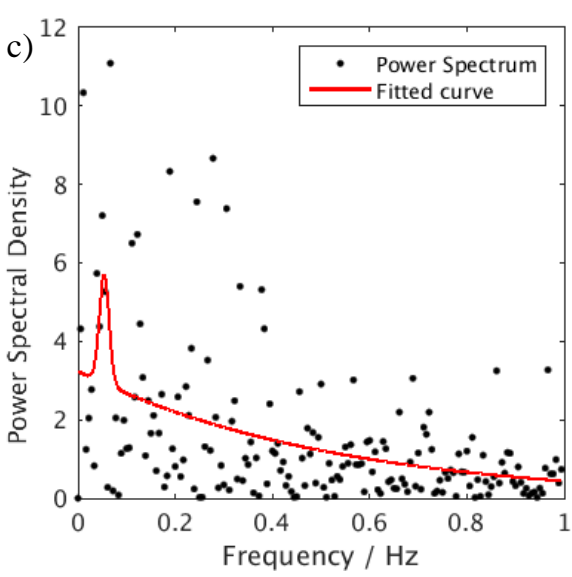

Figure 3 One example FDG subject without respiratory signals detected in COD: a) raw COD trace 3-min tracer, b) Power spectrum of COD, and c) Power spectrum with fitted Gaussian model.

The same spectral analysis was applied to the PCA traces and the same three subjects with measurable respiratory signals were identified. See supplemental materials for examples of PCA spectral analysis results.

For the three identified subjects, the processed COD and PCA signals are shown in Figure 4. Visually, both COD and PCA showed an excellent match with Anzai traces, with COD showing a less accurate match in several inspiration peaks with Anzai than PCA in subject 1. From Table 1, in 2 out of 3 subjects, PCA showed higher correlation coefficients with Anzai than COD (higher by about $10 \%)$. For subject 3, both COD and PCA showed high correlation with Anzai $(\geq 0.9)$. For all three subjects, COD and PCA have high correlation with each other $(\geq 0.85)$. For subject 2, whose correlation coefficients of COD and PCA are only acceptable, with values of 0.63 and 0.71, but have a high COD-PCA correlation of 0.91. In this case, as can be seen from Figure 4, the Anzai trace in black showed saturation at most cycles around end-expiration phase. This may 
lead to the lower correlation coefficients between data-driven signals and Anzai, since Anzai was not providing accurate motion information.

1

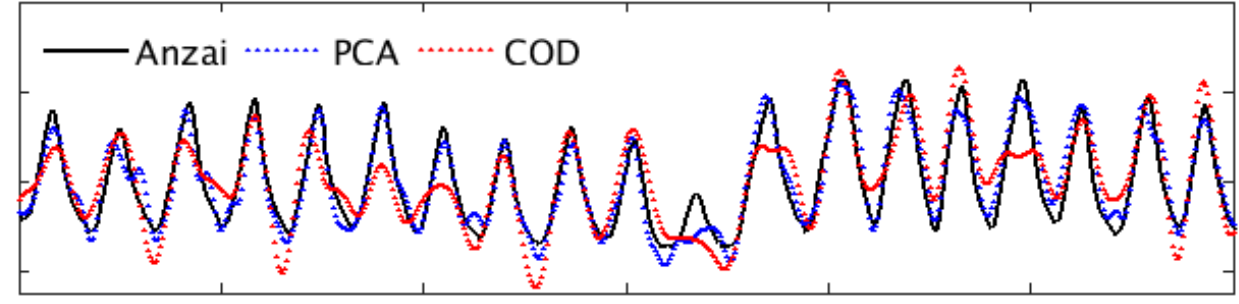

2

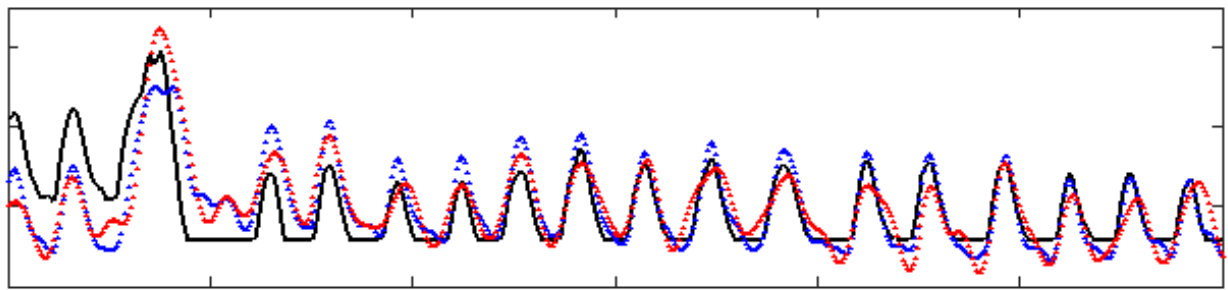

3

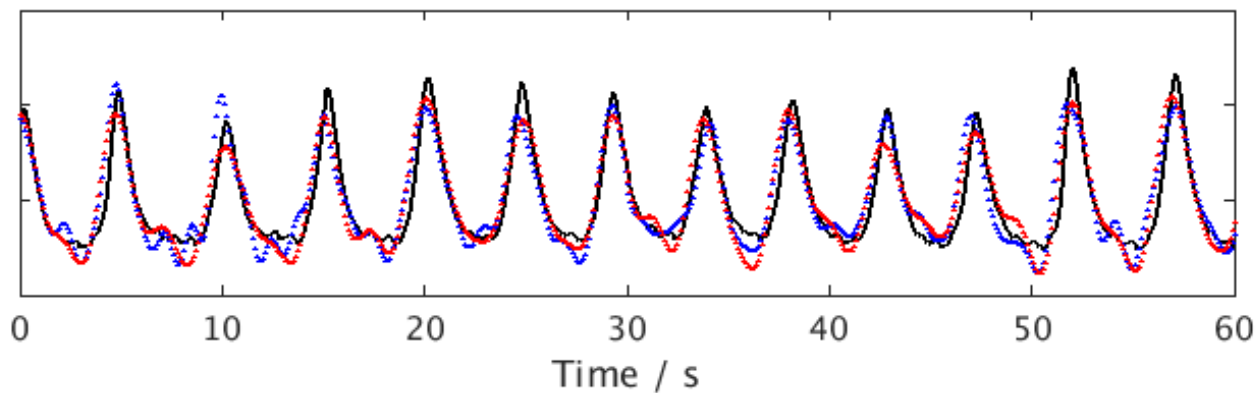

Figure 4 Overlaid standardized Anzai (black), PCA (blue), and COD (red) traces for three ${ }^{18} \mathrm{~F}$ FDG whole body oncology studies displaying $1 \mathrm{~min}$ of data starting $91 \mathrm{~min}$ post-injection. Correlation coefficients of PCA vs. Anzai were: 0.94, 0.75, and 0.93; COD vs. Anzai: 0.84, 0.67, and 0.94; PCA vs. COD: 0.84, 0.89, and 0.95.

\subsection{3. $\quad{ }^{18}$ F-FDG Lung Cancer Studies}

Spectral analysis was also applied to these 10 subjects. Based on the Gaussian fitting results of both COD and PCA spectra, three subjects were identified with measurable respiratory signals. Figure 5 shows a 1-min segment of COD and PCA tracers overlaid with Anzai. For subjects 1 and 2, both COD and PCA showed good correlation with Anzai. However, for subject 3, although 
COD matched very well with PCA, for the first $45 \mathrm{~s}$, both data-driven signals did not correlate with Anzai. Correlation between data-driven and Anzai was as low as 0.21 , but with a high correlation between COD and PCA of 0.79. In this period, the Anzai tracer had 2 peaks for each signal inspiration peaks in the COD and PCA respiratory cycles. For the last $15 \mathrm{~s}$, Anzai showed a very different pattern, with a sharp inspiration peak followed by a long end-expiration phase. In this case, it is not clear whether the Anzai signals are due to irregular breathing patterns or other sources of unreliability compared with the data-driven traces, which seemed to provide more reasonable respiratory motion information. See section 4 for additional discussion.

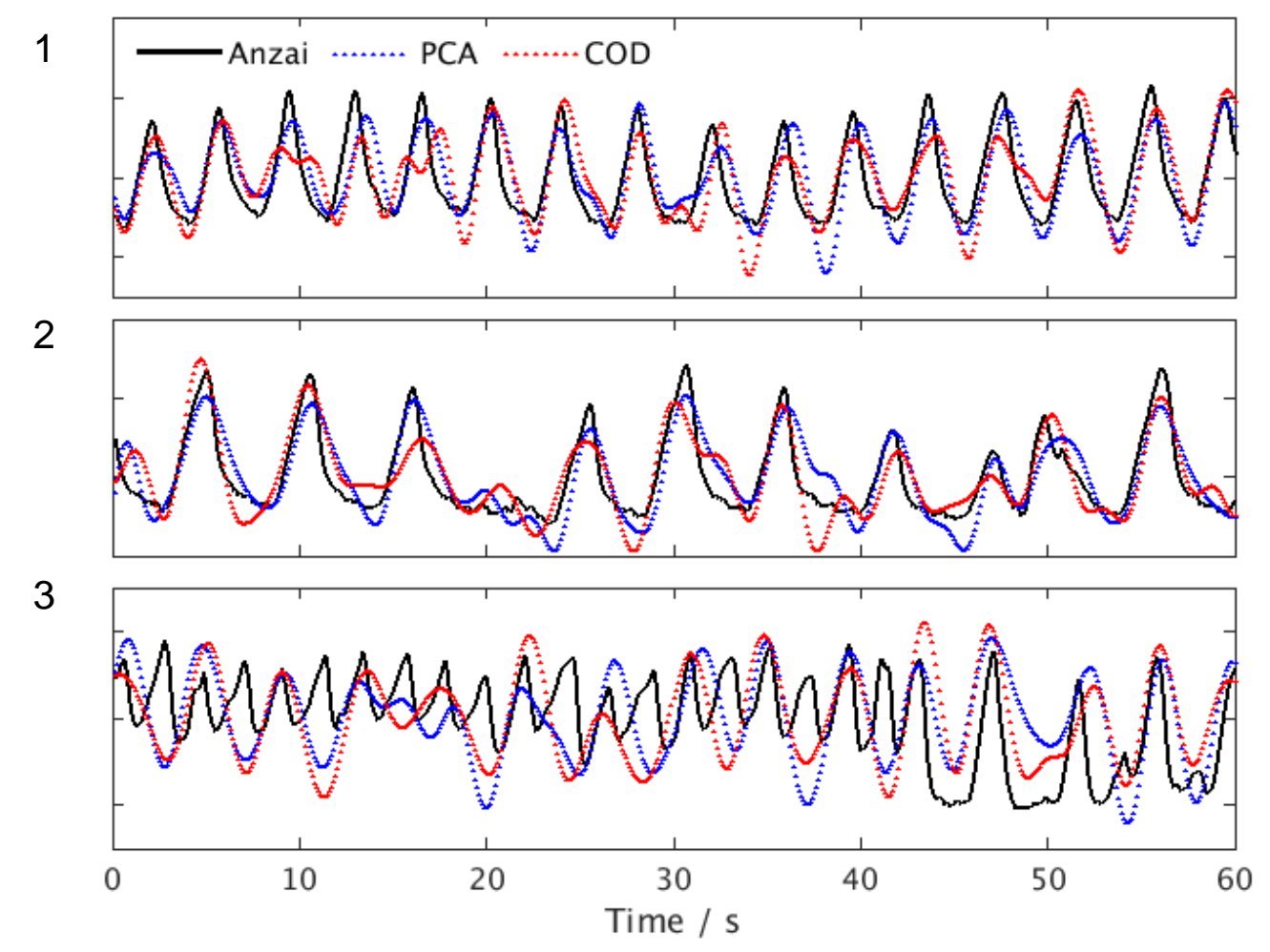

Figure 5 Overlaid standardized Anzai (black), PCA (blue), and COD (red) traces for three ${ }^{18} \mathrm{~F}$ FDG lung cancer studies, displaying $1 \mathrm{~min}$ of data starting $50 \mathrm{~min}$ post-injection. Correlation coefficients of PCA vs. Anzai shown in this figure were: 0.75, 0.85, and 0.22; COD vs. Anzai: $0.69,0.83$, and 0.21 ; PCA vs. COD: $0.82,0.80$, and 0.79 . 


\subsection{Non-Rigid Event-By-Event Respiratory Motion Correction}

\subsection{1. $\quad{ }^{18}$ F-FPDTBZ Pancreas Studies}

Data-driven event-by-event non-rigid motion correction (DD-EBE-NRMC) was applied to three ${ }^{18}$ F-FPDTBZ pancreas studies, using either extracted COD or PCA signals as motion input data instead of Anzai. For comparison purposes, Anzai-based EBE-NRMC was performed, along with reconstructions with no motion correction (NoMC).

To visually compare motion correction results, one coronal slice of each subject is shown in Figure 6. Visually from all three subjects, COD-, PCA-based DD-EBE-NRMC generated comparable results with the Anzai-based method. All three methods showed improved contrast near moving organ edges, such as pancreas, compared with reconstructions with no motion correction. For other tissues than pancreas, a sharper boundary or clearer structures were also observed, such as cold regions within liver (gallbladder and bile duct in subject 2), and a highactivity spot (intestines in subject 3).

For further comparison, line profiles are plotted along a vertical line across the pancreas tail for each subject in Figure 7. As with the visual comparison, line profiles of Anzai, COD, PCA are very similar for these subjects, all having a higher peak activity than the reconstruction without motion correction. The NoMC case also showed a spatial shift in the $\mathrm{Z}$ direction. Therefore, for ${ }^{18}$ F-FPDTBZ pancreas studies, both COD and PCA-based EBE-NRMC showed comparable motion correction results with Anzai, with improved contrast near moving organ edges. 

(a) Anzai
(b) COD
(c) PCA
(d) NoMC

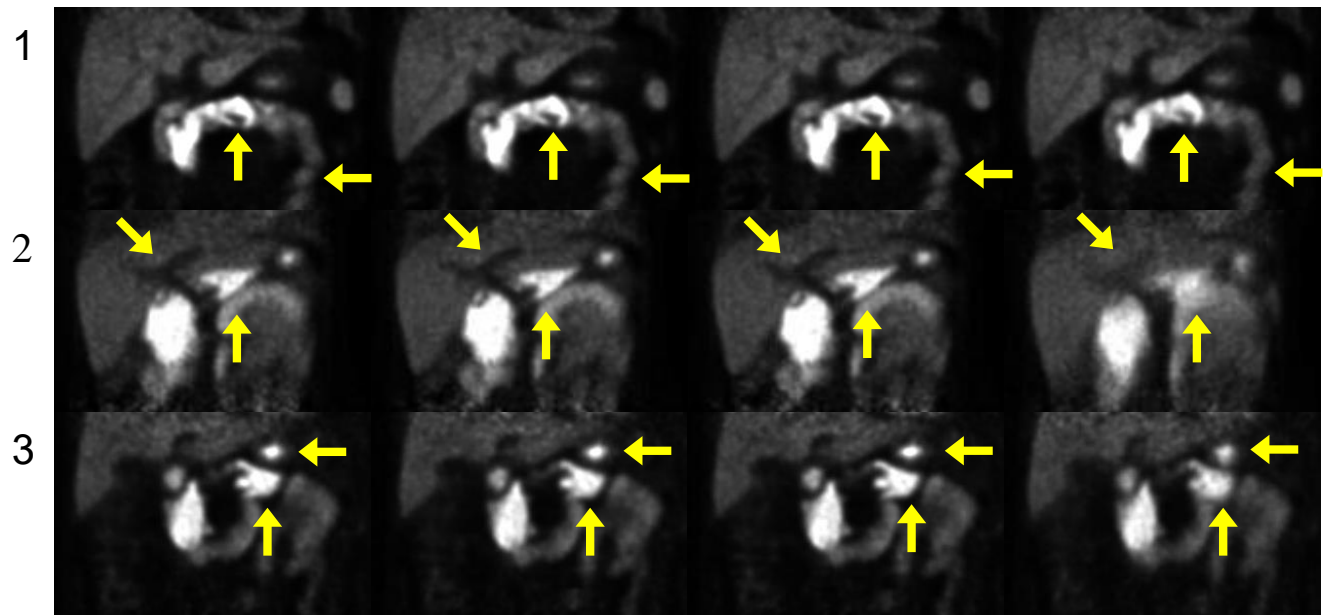

Figure 6 One coronal slice from reconstructions with (a) Anzai, (b) COD, or (c) PCA -based EBE-NRMC, and (d) NoMC, from three ${ }^{18}$ F-FPDTBZ pancreas study subjects. 

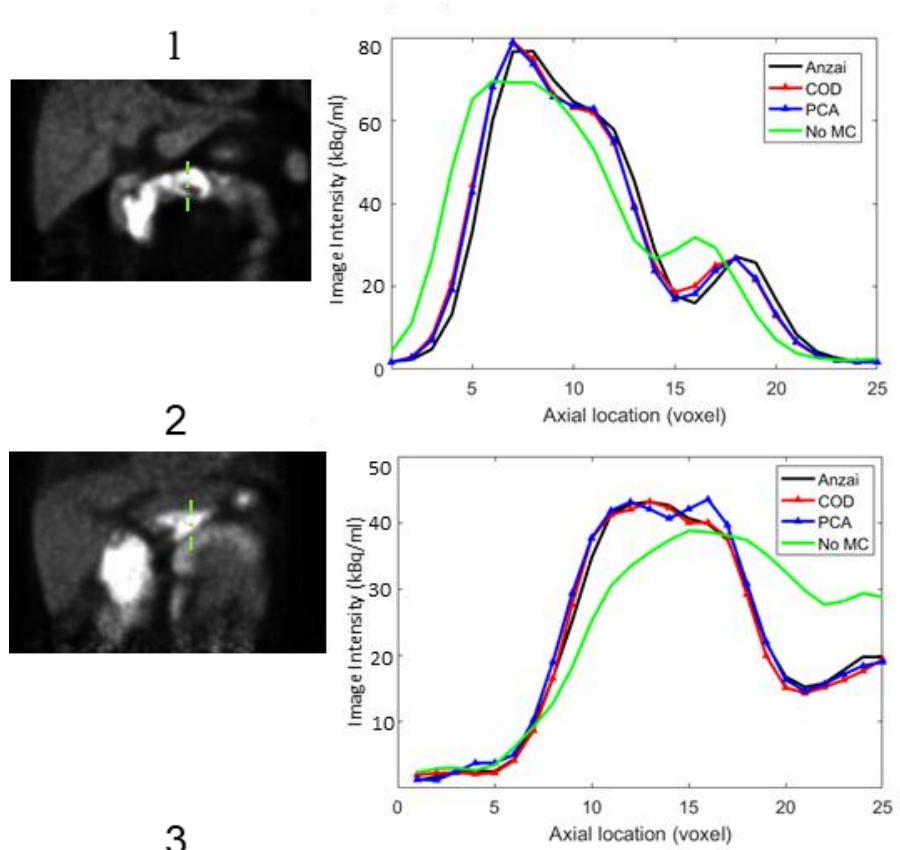

3
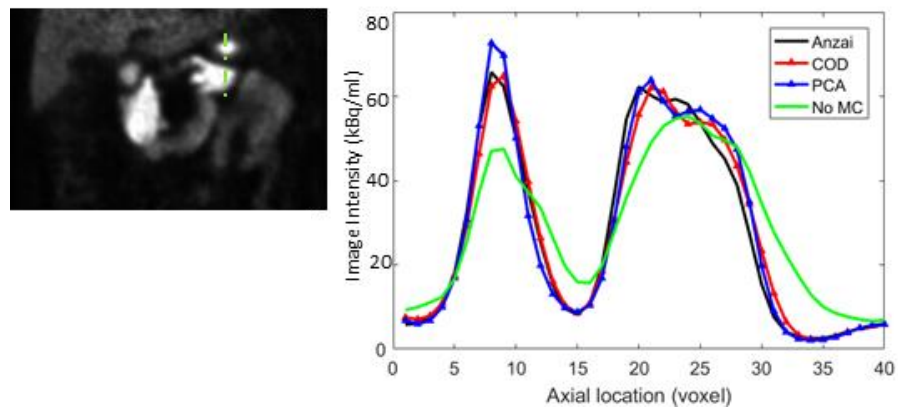

Figure 7 Intensity profiles (right) along a vertical line across the pancreas, as plotted in green on the coronal slice of each subject (left), were extracted from four reconstructions Anzai, COD, PCA -based EBE-NRMC, and No motion correction (NoMC).

\subsection{2. $\quad{ }^{18}$ F-FDG Whole Body Oncology Studies}

For the three identified ${ }^{18} \mathrm{~F}-\mathrm{FDG}$ whole body studies, one slice of each reconstruction with line intensity profiles over lesions for subjects 1,2, and 3 are shown in Figure 8, Figure 9, and Figure 10, respectively. In each case, one slice from reconstructions with (a) Anzai-, (b) COD-, and (c) PCA-based EBE-NRMC results, and (d) reconstruction with no motion correction (NoMC) is shown. For subject 1, with two lesions in the center, all three NRMC results show increased 
image intensity and contrast than the NoMC case, with minor differences between methods. For further comparison, line intensity profiles over the lesion are plotted (Figure 8, right) showing that all three NRMC methods provided comparable results, and led to a much higher intensity (increased by $>100 \%$ ) than NoMC.

For subject 2, one coronal slice is shown in Figure 9, and the small lesion was selected for comparison; a larger tumor is present, however, respiratory motion has smaller effects on larger tumors. Visually, both data-driven methods generated comparable results with the Anzai-based method, with much increased lesion activity. On the right, line profiles showed that the PCA method showed the highest intensity, followed by Anzai and COD. All three methods showed $50 \%$ higher intensity, with a spatial shift in the $\mathrm{Z}$ direction compared to the NoMC case.

For subject 3, one sagittal slice with multiple lung lesions is shown in Figure 10. Two vertically contiguous lesions (see arrow) were not separated clearly in the no-motion correction case. The between-lesion gap was restored for all other methods, with best separation provide by Anzai, followed by COD, and then PCA. Profiles across these two lesions are consistent with the visual interpretation, with peak-to-valley ratio of $37 \%, 47 \%, 62 \%$, and $77 \%$, for Anzai, COD, PCA, and NoMC, respectively. 

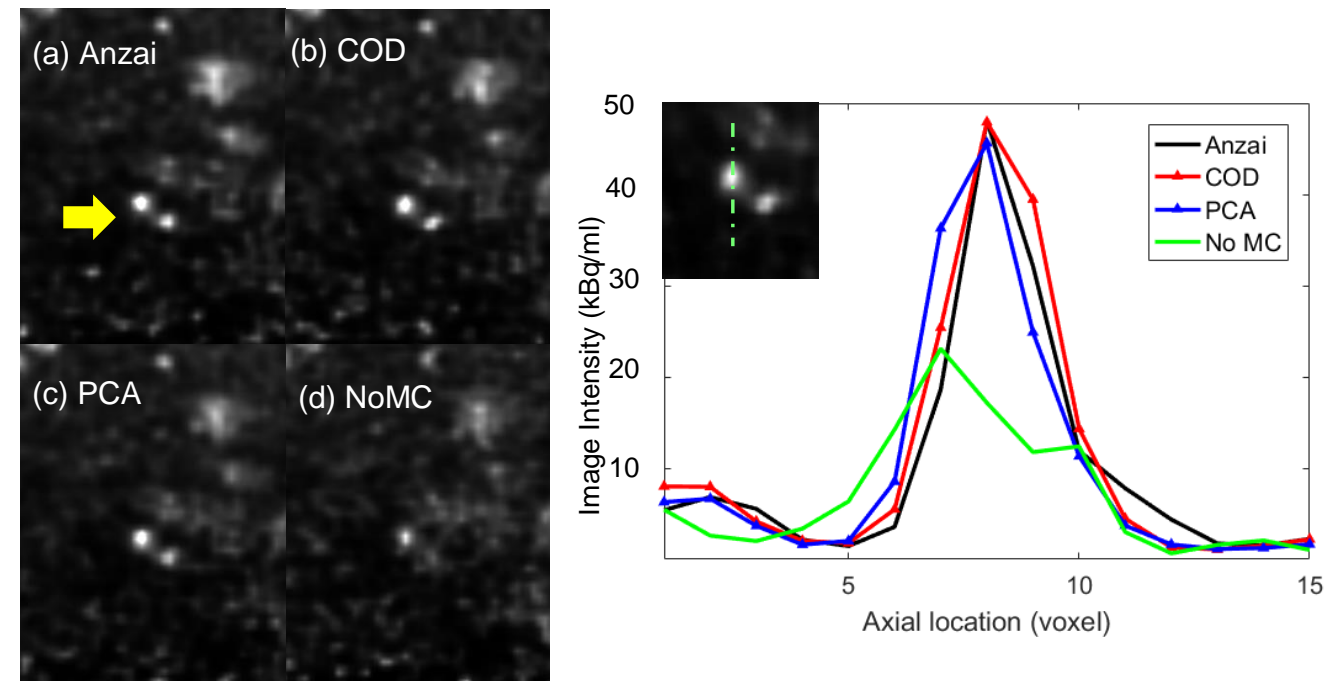

Figure 8 Left: One coronal slice of ${ }^{18} \mathrm{~F}-\mathrm{FDG}$ subject 1 from reconstructions with (a) Anzai-based, (b) CODbased, (c) PCA-based EBE-NRMC, and (d) no motion correction (NoMC). Right: Intensity profiles are shown along the vertical line through the lesion denoted by the arrow in (a).

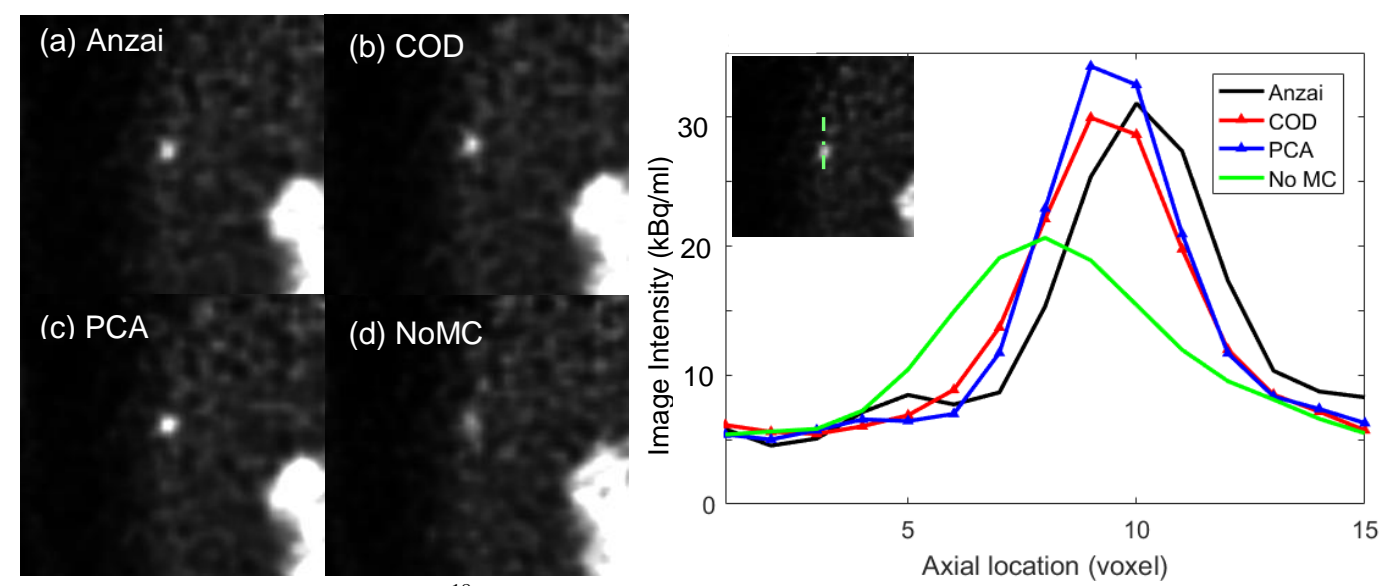

Figure 9 Left: One coronal slice of ${ }^{18}$ F-FDG subject 2 from reconstructions with (a) Anzai-based, (b) CODbased, (c) PCA-based EBE-NRMC, and (d) no motion correction (NoMC), showing a large lesion on the right and a small lesion in the center. Right: Vertical line intensity profiles across the small lesion. 

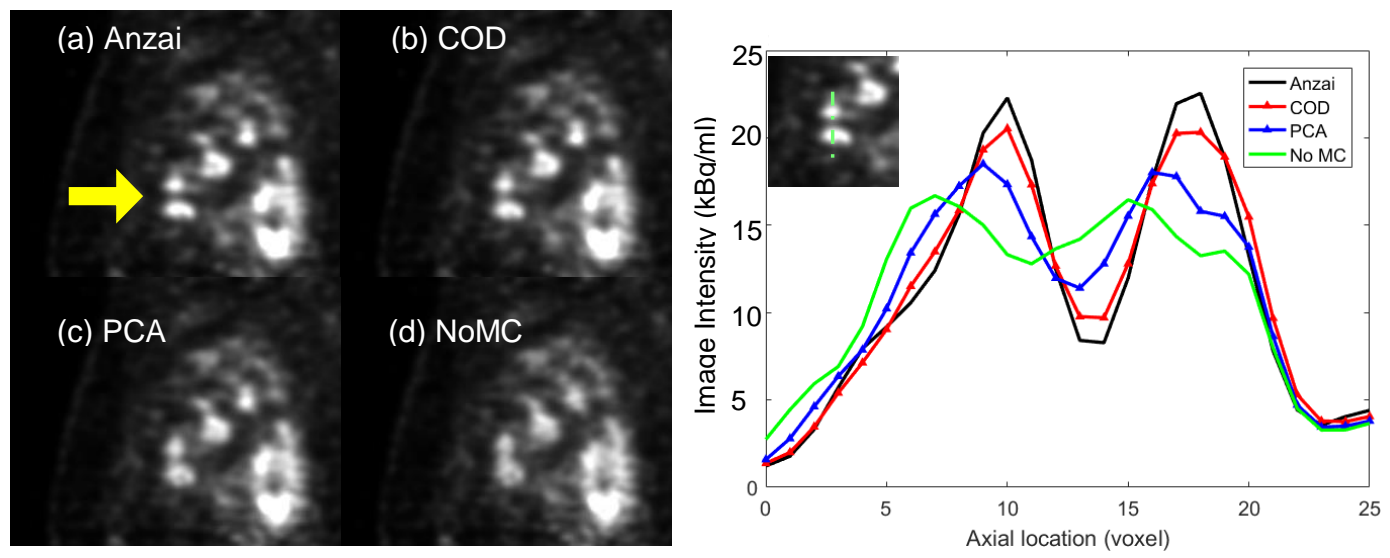

Figure 10 Left: One sagittal slice of ${ }^{18} \mathrm{~F}$-FDG subject 3 from reconstructions with (a) Anzai-based, (b) CODbased, (c) PCA-based EBE-NRMC, and (d) no motion correction (NoMC), showing several lung lesions. Right: Vertical line intensity profiles across two vertically contiguous lesions as denoted by the arrow in (a)

\subsection{3. $\quad{ }^{18}$ F-FDG Lung Cancer Studies}

For the three identified ${ }^{18}$ F-FDG lung cancer studies, sagittal slices are shown in Figure 11. All images are zoomed to show one lesion. In sagittal view, respiration causes motion in the top-left to bottom-right direction. With no motion correction, lesions may have a "tail" in this direction, as is clear for subject 1 (d). For subject 2, the Anzai images showed the least distortion of lesion, with COD and PCA showing some diagonal elongation, but much less distortion than the NoMC images.

To quantitatively analyze motion correction performance, an ROI $\left(4 \times 4 \times 4 \mathrm{~mm}^{3}\right)$ was placed on each lesion, and the \%increase of mean ROI values between Anzai, COD, or PCA compared to NoMC are shown in Table 2. For subject 1, both data-driven methods showed comparable increase in lesion activity as Anzai. For subject 2, all three methods provided improved lesion contrast, with Anzai having the highest increase, followed by PCA, then COD. For subject 3, the subject with irregular Anzai signals (Figure 5), visually, the Anzai images had a slightly larger 
greater distortion than NoMC. Both data-driven methods showed higher lesion intensity (Table 2) with less diagonal elongation (See section 4 for additional discussion of this case).

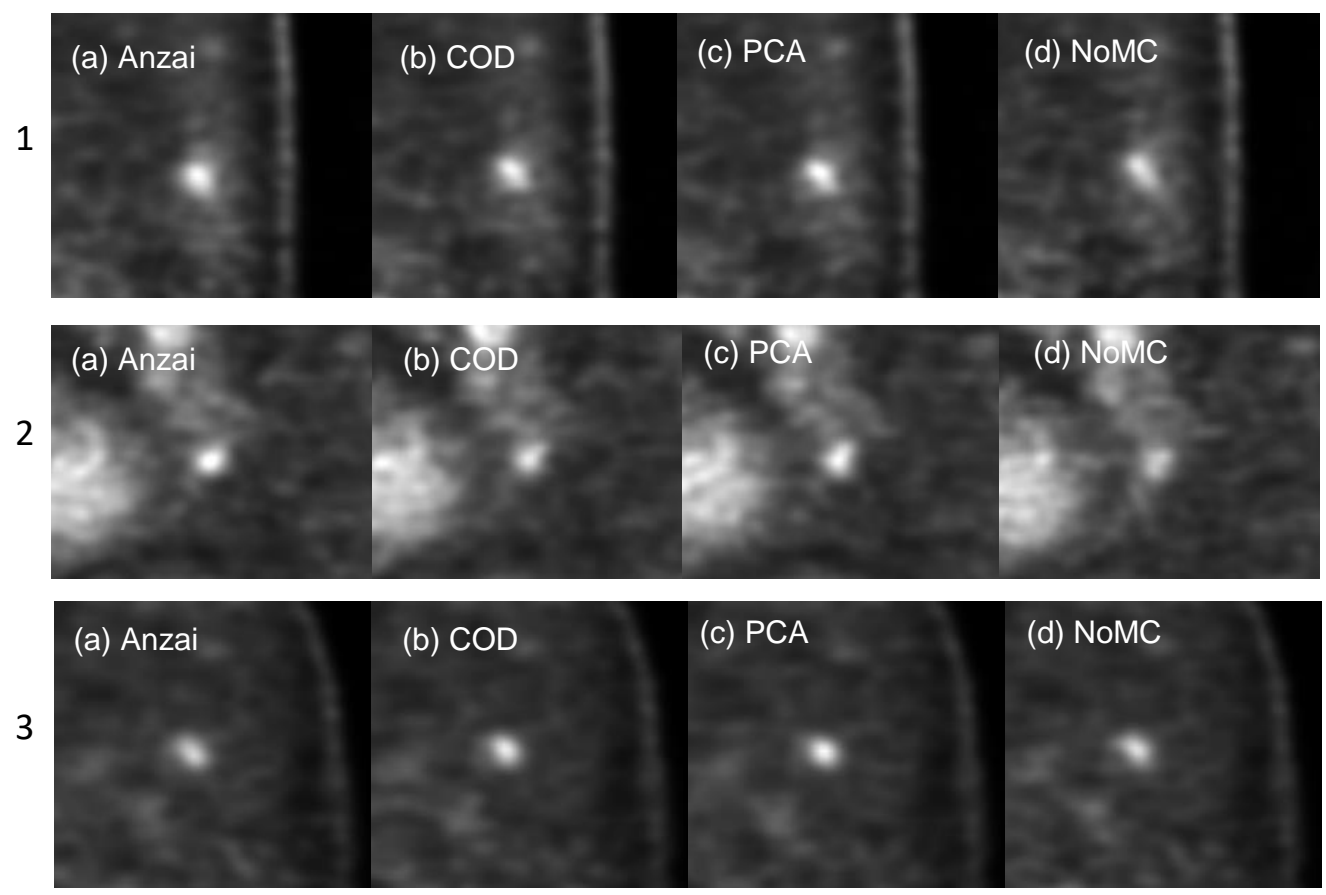

Figure 11 Sample sagittal slices of ${ }^{18}$ F-FDG lung cancer studies 1, 2, and 3. (a), (b) and (c) show EBE-NRMC results with Anzai, COD, and PCA-based respiratory signals, respectively, with (d) showing reconstruction results with no motion correction (NoMC).

Table 2 Quantitative comparison of three ${ }^{18} \mathrm{~F}$-FDG lung cancer subjects. Percent increases in ROI intensity compare to no motion correction (NoMC) are shown.

\begin{tabular}{|c|c|c|c|}
\hline \multirow{2}{*}{ Subject } & \multicolumn{3}{|c|}{ \%increase } \\
\cline { 2 - 4 } & ANZAI & COD & PCA \\
\hline 1 & $13 \%$ & $9 \%$ & $13 \%$ \\
\hline 2 & $38 \%$ & $18 \%$ & $26 \%$ \\
\hline 3 & $-4 \%$ & $7 \%$ & $12 \%$ \\
\hline
\end{tabular}




\section{Discussion}

In this study, we compared two data-driven respiratory motion detection methods: COD and PCA, using Anzai as the gold standard, based on two tracers and three datasets: ${ }^{18} \mathrm{~F}$-FPDTBZ pancreas studies, ${ }^{18} \mathrm{~F}-\mathrm{FDG}$ whole body oncology studies with one bed position over chest or abdomen area, and ${ }^{18} \mathrm{~F}$-FDG lung cancer studies. Then, data-driven event-by-event nonrigid respiratory motion correction (DD-EBE-NRMC) was performed based on either COD- or PCA-based signals. In our previous study [13], COD was applied to ten ${ }^{18} \mathrm{~F}$-FPDTBZ pancreas studies with EBE-rigid-MC. Therefore, in this study, COD was further evaluated on studies with ${ }^{18} \mathrm{~F}-\mathrm{FDG}$, one of the most widely used tracers. In previous studies with PCA [10, 12, 21], the methods were evaluated by ${ }^{18}$ F-FDG studies with gated reconstructions performed. Additionally, the establishment of DDEBE-NRMC based on either COD or PCA showed the utility of data-driven respiratory detection and correction methods.

The selected time frame for the pancreas study was 10-20 min postinjection. Earlier data postinjection were not evaluated here because tracer distribution changes quickly during the first 5 minutes and these changes can substantially affect data-driven traces. For PCA, the tracer dynamics could dominate the respiratory motion, so that the principal components will detect tracer distribution change. For COD, mean tracer distribution change can be removed, however, there may not be enough counts to generate reliable respiratory motion signals. In addition, for this tracer, 10-20 min postinjection is a time period when both tracer uptake in all relevant organs (pancreas, spleen, and kidneys) is high, leading to good image contrast between tissues and 
background. For that same reason, the ${ }^{18} \mathrm{~F}-\mathrm{FDG}$ lung cancer studies, a later stage of data (60-70 min postinjection) was selected for a high tracer uptake in lesions.

For both raw COD and PCA signals, a low-pass filter with $0.5 \mathrm{~Hz}$ cutoff frequency was applied to remove high frequency noise. This cutoff window will not affect respiratory signals as fast as $2 \mathrm{~s} /$ cycle. Although it is rare, some subjects may have irregular breathing with frequencies $\geqslant 0.5$ Hz. If ultra-fast breaths happen in short periods, the low-pass filter would erase those components from the data. A short period of inaccurate respiratory signals is likely to have a minor effect on motion correction results, depending on the total length of study. However, considering the tradeoff between noise and rare irregularly fast cycles, $0.5 \mathrm{~Hz}$ [11] was chosen to be applied for all datasets. Note that short periods of such irregular breathing could also be eliminated from the EBE reconstruction.

When comparing COD with PCA, for most studies, PCA showed higher correlations with Anzai than COD. For the other cases, both COD and PCA had excellent correlation $(\geq 0.9)$ with Anzai. Other than subject 3 in the ${ }^{18} \mathrm{~F}$-FDG lung cancer study with irregular Anzai patterns, both COD and PCA showed good correlation with Anzai, with mean COD-Anzai correlation of $0.81 \pm 0.10$, and mean PCA-Anzai correlation of $0.86 \pm 0.09$. Some of this difference could simply be due to different signal-to-noise levels of the COD and PCA signals. Specifically, the PCA respiratory component is based on the part of the data which shows temporal variation. COD uses all the data, which includes data from portions of the FOV without motion; this subset of data just adds noise. 
There were two cases with a much higher correlation between COD and PCA than between the data-driven methods and Anzai: subject 2 in the ${ }^{18} \mathrm{~F}-\mathrm{FDG}$ WB study, and subject 3 in the ${ }^{18} \mathrm{~F}$ FDG lung study. In the first case, as discussed in 3.1.2, the saturation of Anzai signals resulted in a low correlation between data-driven signals and Anzai. For subject 3 in the lung study (section 3.1.3), both data-driven traces had poor correlation with Anzai (0.16 and 0.19), but COD and PCA had a 0.76 correlation during $60-70$ min postinjection. As described in section 3.1 .3 , the Anzai data showed an irregular pattern of "double peaks". To further test the reliability of Anzai with this type of pattern, EBE-NRMC using Anzai was performed on two 3-min segments of data within 60 to 90 min postinjection, with Anzai of Segment 1 not containing "double peaks", as shown in Figure 12, and Anzai of Segment 2 showing this pattern. For comparison with Anzai, NRMC based on COD or PCA, and NoMC was also performed for these two data segments. A small ROI $\left(4 \times 4 \times 4 \mathrm{~mm}^{3}\right)$ over the lesion was applied to determine the \%increase of mean ROI values between Anzai, COD, or PCA compared to NoMC as shown in Table 3. For Segment 1, without "double peaks", all NRMC methods showed an increase in lesion uptake, as expected. For Segment 2, with the "double peak" pattern, Anzai-based NRMC showed a decrease in lesion uptake, consistent with Table 2, while COD and PCA-based NRMC showing an increase again. Based on these results, it appears that the "double peak" pattern in the Anzai was inaccurate. Data-driven approaches of COD and PCA, reflecting internal motions, provide more reliable respiratory signals. 


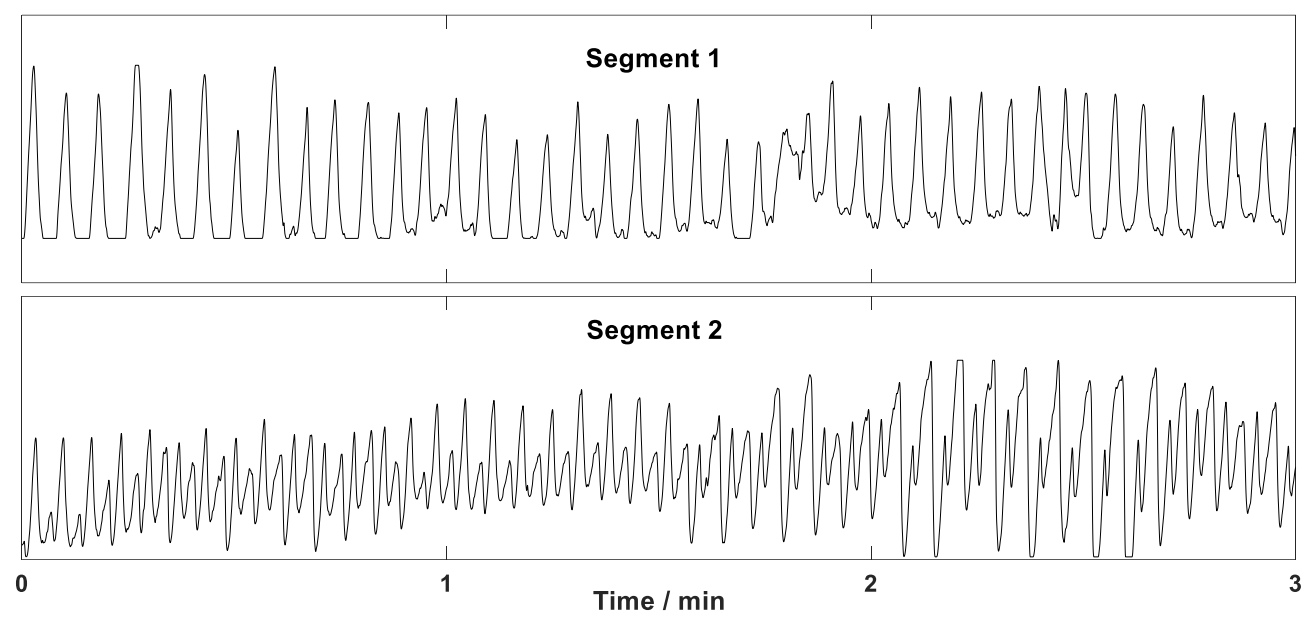

Figure 12 Anzai traces of two 3-min segments from ${ }^{18} \mathrm{~F}$-FDG lung cancer subject 3, with Segment 1 having no "double peaks", and Segment 2 having "double peaks".

Table 3 Quantitative comparison of two 3-min segments of ${ }^{18}$ F-FDG lung cancer subject 3. Percent increases in ROI intensity compare to no motion correction (NoMC) are shown.

\begin{tabular}{|c|c|c|c|}
\hline \multirow{2}{*}{ Segment } & \multicolumn{3}{|c|}{ \%increase } \\
\cline { 2 - 4 } & ANZAI & COD & PCA \\
\hline 1 & $14 \%$ & $6 \%$ & $24 \%$ \\
\hline 2 & $-2 \%$ & $8 \%$ & $7 \%$ \\
\hline
\end{tabular}


EBE-NRMC is dependent on the input respiratory motion trace, as well as the nonrigid registration of reconstructed images between each gate and the reference gate. Control point spacing is one of the most essential parameters of this registration. Smaller spacing may provide more accurate registration, taking more local movement into consideration. However, if the spacing is too fine, especially for noisy images, registrations may become unstable. Therefore, for high-count images, such as the ${ }^{18} \mathrm{~F}$-FPDTBZ studies, a smaller spacing can be chosen for accurate results; for lower-count images, such as the ${ }^{18} \mathrm{~F}-\mathrm{FDG}$ lung studies, a large spacing can be chosen for robust registration results, as discussed in [15]. Another factor to be considered is anatomy. For an organ or a group of organs with similar respiratory movement (such as liver, pancreas, and spleen), a large spacing can be used. For organs such as lung, different locations could have very different respiratory motion. Thus, for lung oncology studies, a smaller control point spacing should be used. In this study, we empirically used $30 \mathrm{~mm}$ for the ${ }^{18} \mathrm{~F}$-FPDTBZ pancreas studies and ${ }^{18} \mathrm{~F}$-FDG liver studies, and $20 \mathrm{~mm}$ for the ${ }^{18} \mathrm{~F}$-FDG lung cancer studies. A more comprehensive study of inter-gate registration parameters is of interest.

In this study, PCA was determined without using TOF information. In a recent study, TOF information was included as one extra dimension of short-frame sinograms (500 ms per frame) [21]. For ${ }^{18} \mathrm{~F}-\mathrm{FDG}$ oncology datasets, TOF-PCA was shown to have increased correlation with signals from the external device. Thus, in future studies, it is desirable to apply TOF-PCA to more datasets to extract improved respiratory signals. In the current study, COD has a lower correlation with Anzai than PCA, but COD-based DD-EBE-NRMC provided comparable results to PCA. Thus, we expect that TOF-PCA, with improved data-driven traces, would provide comparable or slightly better motion-corrected reconstructions. 
In this study, we only used the COD signal in the SI direction. Employing COD signals in the Y direction $\left(\mathrm{C}_{\mathrm{y}}\right)$ together with $\mathrm{C}_{\mathrm{z}}$ for EBE-NRMC could potentially improve motion correction performance. In our previous COD-based DD-EBE-rigid-MC study [13], linear correlations between internal organ motion and data-driven respiratory signals in $\mathrm{Z}$ and $\mathrm{Y}$ directions were built separately using $\mathrm{C}_{\mathrm{z}}$ and $\mathrm{C}_{\mathrm{y}}$, respectively. Like in the previous study, $\mathrm{C}_{\mathrm{y}}$ also provided respiratory motion estimates, but with higher noise than $\mathrm{C}_{\mathrm{z}}$. More evaluation is needed here since, intrinsically, $\mathrm{C}_{\mathrm{y}}$ represents respiratory motion of internal organs in the $\mathrm{Y}$ direction, potentially providing data that could be combined with $\mathrm{C}_{\mathrm{z}}$ to potentially improve EBE-NRMC performance.

Although both COD and PCA detected respiratory motion for the three datasets employed, there are limitations for both methods in terms of tracers and studies. Data-driven methods rely on moving activities in the FOV. COD follows the centroid of all events in each time frame, averaging the motion of all events in the FOV. Therefore, without some concentration of highuptake regions that move with respiration, reliable COD signals cannot be acquired. For all the lung studies that were identified to have respiratory signals based on spectral analysis of either raw COD or PCA, a significant portion of the liver, with high tracer uptake, was in the FOV. For lung studies without data-driven respiratory signals, either little or none of the liver was in the FOV, or the liver had low tracer uptake. For PCA, which extracts "changes" in the data, regions that move are likely also needed to detect respiratory signals, although changes in tissue density could be sufficient[22]. Based on the studies analyzed, PCA provided respiratory motion signals with a higher correlation with Anzai than COD (see Table 1), and less noise than COD (see Figure 4 and Figure 5), which indicates that PCA is more robust than COD in providing respiratory motion signals. However, based on the DD-EBE-NRMC results, COD provided comparable MC results with both PCA and Anzai in ${ }^{18}$ F-FPDTBZ pancreas and ${ }^{18}$ F-FDG whole body studies. In 
the lung cancer studies, PCA-based method generated slightly higher \%increases in SUV than COD. Overall, COD can achieve good performance in respiratory motion estimation and correction and has an advantage in terms of simplicity in implementation and computation.

In addition to respiratory motion detection, COD also able to detect body motion, both for the chest/abdomen area [23], and for brain [24]. By calculating lower frequency COD signals (1s/frame), body motion signals can be detected. Interestingly, the body motion data can be best found in $\mathrm{COD}_{\mathrm{x}}$, the dimension without respiratory information. By extracting sinograms at 1s/frame, PCA may also be able to detect head or body motion[25, 26], although, since PCA provides signals with arbitrary scale, it may be more difficult to assess the magnitude of body motion. COD signals have physical meaning, thus assessing the magnitude of body motion is more straightforward. In the future, it is desirable to incorporate data-driven body motion detection with respiratory motion, to achieve simultaneous EBE motion correction.

\section{Conclusion}

We compared two data-driven respiratory motion estimation methods: COD and PCA, using three datasets with two tracers: ${ }^{18} \mathrm{~F}-\mathrm{FPDTBZ}$, and ${ }^{18} \mathrm{~F}-\mathrm{FDG}$. For all studies, except one with unusual Anzai traces, both COD and PCA showed good-to-excellent correlation with Anzai, with PCA showing higher correlations with Anzai than COD in most cases. We further developed a data-driven event-by-event nonrigid respiratory motion correction method based on either COD or PCA-derived respiratory signals. COD-based NRMC showed comparable motion correction results as those of PCA, both of which were comparable with Anzai-based results. All methods 
showed great reductions in motion-induced blurring compared to reconstructions without motion correction. Therefore, for many imaging situations, both COD and PCA are able to provide reliable data-driven respiratory motion estimates for data-driven event-by-event non-rigid motion correction.

\section{References}

[1] D. Visvikis, O. Barret, T. Fryer, A. Turzo, F. Lamare, C. C. L. Rest, et al., "A posteriori respiratory motion gating of dynamic PET images," in 2003 IEEE Nuclear Science Symposium. Conference Record (IEEE Cat. No.03CH37515), 2003, pp. 32763280 Vol.5.

[2] R. A. Bundschuh, A. Martinez-Moeller, M. Essler, M. J. Martinez, S. G. Nekolla, S. I. Ziegler, et al., "Postacquisition detection of tumor motion in the lung and upper abdomen using list-mode PET data: A feasibility study," Journal of Nuclear Medicine, vol. 48, pp. 758-763, May 2007.

[3] P. J. Schleyer, M. J. O'Doherty, S. F. Barrington, and P. K. Marsden, "Retrospective data-driven respiratory gating for PET/CT," Physics in Medicine and Biology, vol. 54, pp. 1935-1950, Apr 2009.

[4] P. J. Schleyer, M. J. O'Doherty, and P. K. Marsden, "Extension of a data-driven gating technique to 3D, whole body PET studies," Physics in Medicine and Biology, vol. 56, pp. 3953-3965, Jul 2011.

[5] P. J. Schleyer, K. Thielemans, and P. K. Marsden, "Extracting a respiratory signal from raw dynamic PET data that contain tracer kinetics," Physics in Medicine and Biology, vol. 59, pp. 4345-4356, Aug 2014.

[6] A. L. Kesner, R. A. Bundschuh, N. C. Detorie, M. Dahlbom, S. I. Ziegler, J. Czernin, et al., "Respiratory Gated PET Derived in a Fully Automated Manner From Raw PET Data," Ieee Transactions on Nuclear Science, vol. 56, pp. 677-686, Jun 2009.

[7] A. L. Kesner and C. Kuntner, "A new fast and fully automated software based algorithm for extracting respiratory signal from raw PET data and its comparison to other methods," Medical Physics, vol. 37, pp. 5550-5559, Oct 2010.

[8] J. He, G. J. O'Keefe, S. J. Gong, G. Jones, T. Saunder, A. M. Scott, et al., "A novel method for respiratory motion gated with geometric sensitivity of the scanner in 3D PET," IEEE Transactions on Nuclear Science, vol. 55, pp. 2557-2565, 2008.

[9] F. Buther, I. Ernst, M. Dawood, P. Kraxner, M. Schafers, O. Schober, et al., "Detection of respiratory tumour motion using intrinsic list mode-driven gating in positron emission tomography," European Journal of Nuclear Medicine and Molecular Imaging, vol. 37, pp. 2315-2327, Dec 2010.

[10] K. Thielemans, S. Rathore, F. Engbrant, and P. Razifar, "Device-less gating for PET/CT using PCA," in 2011 IEEE Nuclear Science Symposium Conference Record, 2011, pp. 3904-3910.

[11] K. Thielemans, P. Schleyer, P. K. Marsden, R. M. Manjeshwar, S. D. Wollenweber, and A. Ganin, "Comparison of different methods for data-driven respiratory gating of 
PET data," in 2013 IEEE Nuclear Science Symposium and Medical Imaging Conference (2013 NSS/MIC), 2013, pp. 1-4.

[12] O. Bertolli, S. Arridge, S. D. Wollenweber, C. W. Stearns, B. F. Hutton, and K. Thielemans, "Sign determination methods for the respiratory signal in data-driven PET gating," Physics in Medicine and Biology, vol. 62, p. 3204, 2017.

[13] S. Ren, X. Jin, C. Chan, Y. Jian, T. Mulnix, C. Liu, et al., "Data-driven event-by-event respiratory motion correction using TOF PET list-mode centroid of distribution," Physics in Medicine \& Biology, vol. 62, p. 4741, 2017.

[14] C. Chan, J. Onofrey, Y. Jian, M. Germino, X. Papademetris, R. E. Carson, et al., "Non-rigid Event-by-event Continuous Respiratory Motion Compensated List-mode Reconstruction for PET," IEEE Transactions on Medical Imaging, vol. PP, pp. 1-1, 2017.

[15] Y. Lu, K. Fontaine, T. Mulnix, J. A. Onofrey, S. Ren, V. Y. Panin, et al., "Respiratory Motion Compensation for PET/CT with Motion Information Derived from Matched Attenuation Corrected Gated PET Data," Journal of nuclear Medicine, 2018.

[16] Y. lun Chou, "Statistical analysis," in Holt International, ed, 1975.

[17] K. Thielemans, C. Tsoumpas, S. Mustafovic, T. Beisel, P. Aguiar, N. Dikaios, et al., "STIR: software for tomographic image reconstruction release 2," Physics in Medicine and Biology, vol. 57, p. 867, 2012.

[18] M. D. Normandin, K. F. Petersen, Y. S. Ding, S. F. Lin, S. Naik, K. Fowles, et al., "In Vivo Imaging of Endogenous Pancreatic beta-Cell Mass in Healthy and Type 1 Diabetic Subjects Using F-18-Fluoropropyl-Dihydrotetrabenazine and PET," Journal of Nuclear Medicine, vol. 53, pp. 908-916, Jun 2012.

[19] A. Joshi, D. Scheinost, H. Okuda, D. Belhachemi, I. Murphy, L. H. Staib, et al., "Unified Framework for Development, Deployment and Robust Testing of Neuroimaging Algorithms," Neuroinformatics, vol. 9, pp. 69-84, 2011.

[20] V. Y. Panin, M. Defrise, J. Nuyts, A. Rezaei, and M. E. Casey, "Reconstruction of uniform sensitivity emission image with partially known axial attenuation information in PET-CT scanners," in 2012 IEEE Nuclear Science Symposium and Medical Imaging Conference Record (NSS/MIC), 2012, pp. 2166-2173.

[21] O. Bertolli, S. Arridge, C. W. Stearns, S. D. Wollenweber, B. F. Hutton, and K. Thielemans, "Data driven respiratory signal detection in PET taking advantage of time-of-flight data," in 2016 IEEE Nuclear Science Symposium, Medical Imaging Conference and Room-Temperature Semiconductor Detector Workshop (NSS/MIC/RTSD), 2016, pp. 1-3.

[22] O. Bertolli, V. Cuploy, S. Arridge, C. W. Stearns, S. D. Wollenweber, B. F. Hutton, et al., "Detection of Lung Density Variations with Principal Component Analysis in PET," in IEEE Medical Imaging Conference, Atlanta, GA, USA, 2017.

[23] Y. Lu, S. Ren, M. Naganawa, J. D. Gallezot, K. Fontaine, J. Wu, et al., "Non-rigid event-by-event body motion correction with automated data-driven motion detection for static and dynamic PET," in IEEE Nucl. Sci. Symp. Med. Imag. Conf. Rec., Atlanta, USA, 2017.

[24] Y. Lu, J. D. Gallezot, M. Naganawa, K. Fontaine, T. Toyonaga, S. Ren, et al., "Datadriven motion detection and event-by-event correction for brain PET," in SNMMI, 2018.

[25] K. Thielemans, P. Schleyer, J. Dunn, P. K. Marsden, and R. M. Manjeshwar, "Using PCA to detect head motion from PET list mode data," in Nuclear Science Symposium and Medical Imaging Conference (NSS/MIC), 2013 IEEE, 2013, pp. 1-5. 
[26] P. J. Schleyer, J. T. Dunn, S. Reeves, S. Brownings, P. K. Marsden, and K. Thielemans, "Detecting and estimating head motion in brain PET acquisitions using raw time-of-flight PET data," Phys Med Biol, vol. 60, pp. 6441-58, Aug 212015. 